\title{
Effects of Shadowing on LoRa LPWAN Radio Links
}

\author{
Mohamed Hadi Habaebi, Israth Jahan Chowdhury, Md Rafiqul Islam, Nur Aishah Binti Zainal \\ Department of Electrical and Computer Engineering, International Islamic University Malaysia, Malaysia
}

\begin{tabular}{l} 
Article Info \\
\hline Article history: \\
Received Jan 15, 2017 \\
Revised Aug 9, 2017 \\
Accepted Aug 26, 2017 \\
\hline
\end{tabular}

Keyword:

Low power wide area network long range

Wireless communication internet of things

\begin{abstract}
LoRaWAN is a long-range, low-power, wireless telecommunications method; expected to play a big role for the Internet of Things. End appliances use LoRaWAN through a single wireless hop to communicate with gateways linked to the Internet that function as transparent bridges relaying messages amongst these end-devices and a central network server. This technology youtes a combination of extended range, low power utilization and protected data communication and is gaining significant traction in IoT networks being deployed by wireless network operators. However, no comprehensive evaluation of the technology exists in the open literature. The main intention of this paper is to investigate the effects of shadowing on LoRaWAN links and analyze the performance in terms of packet loss ratio for different physical layer settings. Results indicate large differences in performance when shadowing is taken into consideration upsetting the expected performance tremendously.
\end{abstract}

Copyright $@ 2017$ Institute of Advanced Engineering and Science. All rights reserved.

\section{Corresponding Author:}

Mohamed Hadi Habaebi, Department of Electrical and Computer Engineering, International Islamic University Malaysia, Jalan Gombak, Kuala Lumpur, 53100, Malaysia.

Email: habaebi@iium.edu.my

\section{INTRODUCTION}

The transmission of information or power among two or more points that are not associated by an electrical conductor is wireless communication. Radio is used by the most frequent wireless technologies. Distances can be made shorter with radio waves, as example only some meters for television or as far as thousands or even millions of kilometers. It covers various types of rigid, mobile, and portable applications. Some methods of attaining wireless communications consists the use of other electromagnetic wireless technologies, such as light, magnetic, or electric fields or the making use of sound. Wireless operations consent services, such as long-range communications, that are not possible or impractical to execute with the use of wires [1].

LoRa is a form of low power wide area wireless network protocols for Internet of Thing applications. LoRa means Long Range Radio. It is the proprietary wireless technology developed by Semtech Corporation principally targeted for M2M as well as IoT networks [2]. This technology came with an expectation to facilitate public or multi tenant networks to unite multiple applications running in the same network and to accomplish the hope of developing smart city with the assistance of LoRa sensors and automated products/applications [3]. It utilizes a spread spectrum modulation in the Sub-GHz band to enable long range (greater than 10 miles) coverage, low power consumption (up to 10 years battery power), high network capacity (up to 1 million nodes), robust communication, and localization facility [2].

On March 2, 2015 Microchip Technology Inc., a chief supplier of microcontroller, mixed-signal, analog and Flash-IP solutions, announced the earliest in a series of modules for the LoRa (Long range) wireless networking standard that facilitates Internet of Things (IoT) and Machine-to-Machine (M2M) wireless communication by means of a range of more than 10 miles, a battery life of greater than 10 years, and the facility to tie millions of wireless sensor nodes to LoRa technology gateways. Version 1.0 of the 
LoRaWAN specification, was released on 16 June 2015 from the LoRa Alliance; which illustrates the LoRaWANTM network protocol. LoRaWANTM is a Low Power Wide Area Network (LPWAN) specification intended for wireless things that is functioned using battery in local, national or universal network. This standard will make available seamless interoperability among smart things not including the need of difficult local installations and gives back the liberty to the user, developer, businesses enabling the roll out of Internet of Things [4]. Low-Power Wide Area Network (LPWAN) is a sort of wireless telecommunication network designed to permit long range communications at a small bit rate among things (connected objects), such as sensors operated on a battery.

This paper is organized as follows. Section 2 introduces and describes the architecture of LoRa. Section 3 illustrates the methods of research. Section 4 demonstrates the results and analyzes them. Section 5 concludes the paper and summarizes the opportunities.

\section{LORA TECHNOLOGY}

Generally, a network based on LoRa wireless technology can afford coverage which is superior in range compared to the range of present cellular networks. LoRa intends to enable IoT through the LPWAN standardization by deploying around the world. LoRa technology has its dynamic architecture and lots of dominant features. Here, the first section describes the LoRa architecture and the other shows the transmission constraints of LoRa.

\subsection{Architecture}

LoRaWAN network architecture is characteristically laid out in a star-of-stars topology in which gateway is a see-through link relaying messages among end-devices and a central network server in the backend. Gateways are associated to the network server via standard IP connections whereas end-devices make use of single-hop wireless communication to one or loads of gateways. All end-point communication is generally bi-directional, however they also support operations like multicast enabling software upgrade over the air or other mass distribution messages to decrease the on air communication time [4].

LoRa is a spread spectrum modulation scheme, supported on chirp spread spectrum modulation and utilizes wideband linear frequency modulated pulses whose frequency increases or decreases over a definite quantity of time to instruct information. The main properties of LoRa are: extended range, elevated robustness, multipath resistance, Doppler resistance and low power. The LoRa transceivers, we can find nowa-days are able to function between $137 \mathrm{MHz}$ to $1020 \mathrm{MHz}$, so they can also function in licensed bands. Though, they are frequently installed in ISM bands (EU: $433 \mathrm{MHz}$ and $868 \mathrm{MHz}$, USA: $433 \mathrm{MHz}$ and $915 \mathrm{MHz}$ ). The chip rate as well as the programmed bandwidth (chip-per-second-per-Hertz) are identical and can take values of 125, 250 or 500kHz. Besides, the Spreading Factor for a LoRa link may be wide-ranging depending on the communication distance and preferred on-air time [5].

\subsection{Transmission Constraints}

LoRa has some parameters on hand for its configuration. Such as: Spreading Factor, Carrier Frequency, Transmission Power, Bandwidth and Coding Rate. The evaluation of LoRa will be done using these parameters.

Spreading Factor: The Principle of SF is every bit of information is supposed to be encoded like several chips. The liaison among the bit and chip rate for LoRa modulation is that chip rate is equal to the multiplication of bit rate and 2sf [5]. The relation of just a single bit for multiple chips of information depicts that spreading factor is having direct effect on the length of LoRa packet. SF can be selected from 6 to 12 but only 6 and 12 will be used for this work. The increase in SF increases several constraints like communication range, airtime, SNR as well as the sensitivity of packet.

Carrier Frequency: The broadcasting of a preset frequency that has been altered to carry data and measured in Hertz (cycles per second) is called Carrier Frequency. Here, $868 \mathrm{MHz}$ is being used as Carrier frequency.

Transmission Power: Power transmission is the travelling of power from its place of production to a location somewhere it can be applied to execute useful work. The range of TP for LoRa radio is from $(-4 \mathrm{dBm})$ to $20 \mathrm{dBm}$. It can also be between $2 \mathrm{dBm}$ to $20 \mathrm{dBm}$ depending on the hardware [6].

Bandwidth: BW is the range of frequencies within a given band, in particular that used for transmitting a signal. Selection of Spreading Factor should be supervised depending on the BW. Choosing a narrow BW increases the sensitivity along with the time on air. Increase of BW makes the transmission faster and 
decreases the sensitivity. LoRa modulation drives the data stream on a chip rate which is equivalent to the programmed bandwidth in chips per-second-per-Hertz. So the $125 \mathrm{kHz}$ bandwidth is supposed to correspond to a chip rate of $125 \mathrm{kcps}$. SX1272 is having the programmable bandwidth settings $500 \mathrm{kHz}, 250 \mathrm{kHz}$ and $125 \mathrm{kHz}$ and the $\mathrm{SX} 1276$ has bandwidths from $500 \mathrm{kHz}$ to as low as $7.8 \mathrm{kHz}$ [5].

Coding Rate: CR is the Forward Error Correction rate which is actually needed while bursts of interference can be occurred in the radio link. An elevated CR enhances time on air. CR can be selected between 4/5, 4/6, $4 / 7$ to $4 / 8[5]$.

\section{RESEARCH METHOD}

\subsection{Simulation Platform}

The simulator platform used was developed by the author in [6] as part of their evaluation of LoRa technology. It's a straight forward implementation of the air interface of LoRaWAN and was written in Python. We have modified the sourcecode to suit our study and to further investigate other aspects of LoRaWAN technology, highlight the drawbacks in the design of the air interface and to optimize the performance based on the predefined set of dynamic parameters given in the standard. This is considered the best technique for evaluating the performance of LoRa technology due to its flexibility in presenting the results and correlating the behavior of different players in the technology. In order to benchmark against the work done in [6], we repeated the same experiments done in order to highlight the effect of shadowing on the performance.

\subsection{Path Loss Model}

It is the drop in power density of an electromagnetic wave as it circulates through space. Path loss is a major component in the analysis and design of the link budget of a telecommunication system. This name is frequently used in wireless communications and also in signal propagation. Path loss can happen due to many effects, such as free-space loss, refraction, diffraction, reflection, aperture-medium coupling loss, and absorption. Here, the expected path loss is determined as:

$$
E P L=B+10 n \log 10(d / d 0)+\sigma_{\mathrm{SF}}
$$

where $\mathrm{B}$ is path loss, $\mathrm{n}$ is path loss exponent, $\mathrm{d}$ is the distance among the node and the base station, $\mathrm{d} 0$ is the $1 \mathrm{~km}$ reference distance and $\sigma \mathrm{SF}$ the standard deviation of shadow fading (SF). Table 1 lists the value of path loss exponent, intercept and shadow fading [7]. We have resorted to the model parameters reported in [7] rather than those in [6] due to their realistic values. The propagation model parametrs used was developed for for a city-side coverage range and resembles a typical modern urban city plan.

Table 1. Channel Characteristics

\begin{tabular}{ll}
\hline Metric & Values \\
\hline Path loss exponent(n) & 2.32 \\
Path loss intercept $(B)$ & 128.95 \\
Shadow fading $(\sigma S F)$ & $7.8 \mathrm{~dB}$ \\
\hline
\end{tabular}

\subsection{Evaluation Metrics}

To estimate the performance of LoRa LPWAN technology three metrics are being defined: Data Extraction Rate (DER), Network Energy Consumption (NEC) and percentage of Lost Packets. Each and every transmitted message must be acknowledged by means of the backend system while talking about an effctive LoRa deployment this means that every transmitted message needed to be collected properly by at least one LoRa sink. However, in this study, we shall consider the metric of percentage of lost packets.

Percentage of Lost Packets: Packet loss takes place while one or more packets of data moving across a computer network, becomes unsuccessful to arrive their target. Packet loss is generally caused because of network congestion. As soon as content appear for a sustained period at a particular router or network segment at a rate larger than it is feasible to send through, then there is no other choice left than to drop packets. Packet loss is measured as a percentage of packets lost with respect to packets sent. In this paper, Percentage of Lost Packets are estimated as: 


\section{SIMULATION RESULTS AND ANALYSIS}

In the beginner experiments we assess the average capacity of LoRa using an uncomplicated setup where $\mathrm{N}$ nodes are being transmitted to simply one sink. In these experiments, standardized transmitter configuration set $\mathrm{SN}=\{\mathrm{SF}, \mathrm{CF}, \mathrm{TP}, \mathrm{BW}, \mathrm{CR}\}$ will be used. Nodes are positioned indiscriminately in the region of the sink in a way that all nodes can get to the sink with the prearranged SN setting. Three transmitter configurations SN1, SN2 and SN3 are given in Table 2. In all settings, it is being imagined that a packet of 20byte is being sent by all nodes on every $16.7 \mathrm{~min}$ representing a realistic application. The chosen setting for SN1 is the most strong transmitter settings LoRa which leads to transmissions having the greatest feasible airtime of $1712.13 \mathrm{~ms}$, for SN2; the setting of transmission leads to the smallest airtime of $7.07 \mathrm{~ms}$ and for SN3 the chosen setting is the one which is used by common LoRaWAN deployments. [6]

Table 2. Set of Parameters

\begin{tabular}{|c|c|c|c|}
\hline Parameter & SN1 & SN2 & SN3 \\
\hline Spreading Factor & 12 & 6 & 12 \\
\hline Carrier Frequency & 868 & 868 & 868 \\
\hline Transmit Power & 14 & 14 & 14 \\
\hline Bandwidth & 125 & 500 & 125 \\
\hline Coding Rate & $4 / 8$ & $4 / 5$ & $4 / 5$ \\
\hline
\end{tabular}

SN1 is used with simple channel models and LoRa channel models to examine the impact of these further practical channel representations. All the following experiments are using LoRa channel demonstration. In Figure 1 and Figure 2 the outcome of the first set of experiments are being shown. Every one of the data points represent a simulation run with the increasing number of nodes from 100 to 1600 , percentage of lost packets stays totally same for all the cases while shadowing effects are not used. While shadowing effects are used, the initial communication range is $1 \mathrm{~km}$ for all set of experiments. But when the shadowing effect is being added (Figure 2) for simulation an increasing number of nodes shows constant rise and drop. Most of the results are nearly same.

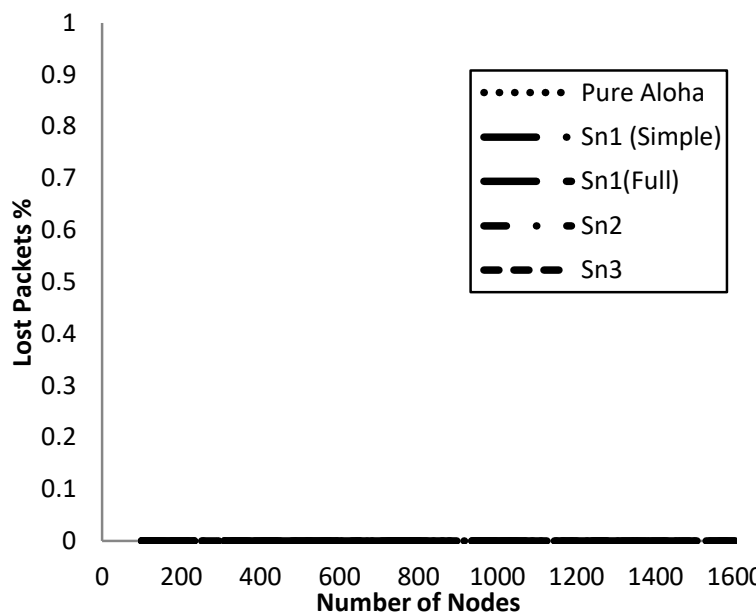

Figure 1. Lost Packets \% (without shadowing effect)

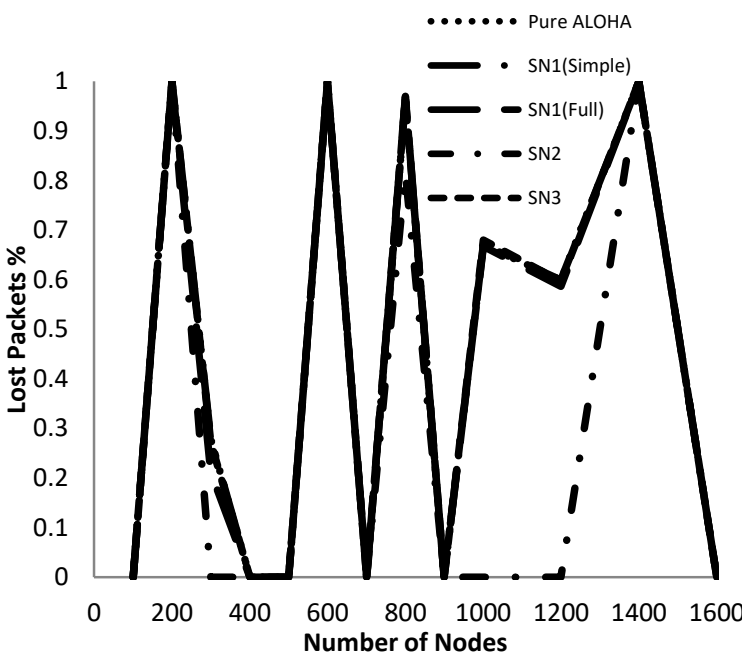

Figure 2. Lost Packets\% (with shadowing effect)

The next set of experiment is evaluated to perceive the impact of dynamic communication parameter selection on Percentage of Lost Packets. Three transmitter configurations: SN3, SN4 and SN5 will be compared and SN3 will be the same as in Experiment Set 1 and being used as reference. For this, the data broadcasting rate has been reduced from a packet of 20 byte each $16.7 \mathrm{~min}$ to each $22 \mathrm{~min}$. Indicating other settings, the assumption will be again a packet of 20 byte sent via every node each $16.7 \mathrm{~min}$ and the Carrier Frequency is $868 \mathrm{MHz}$. N number of nodes are send to one sink. Nodes will be located at random in the 
region of the sink surrounded by a radius that makes sure that every one of the nodes can get to the sink but for this; the most strong settings should be used. On the other hand, for all the nodes the SF, BW, and CR are placed as if the airtime is decreased (SN4 having invariable TP $=14 \mathrm{dBm}$ ) and after that the first airtime and afterward TP is reduced (SN5). The LoRa channel representation is being used for all experiments.

As shown in Figure 3 and Figure 4, the most favorable settings which is related to airtime and airtime plus Transmit Power have enormous impact when talking about reachable DER and lost packets. With reduced airtime (SN4) as well as a minimized airtime plus Transmit Power, 100\% packets are being lost while shadowing effects are not used for calculation. This is just same as the first experiment which is attained with stationary conservative settings the same as used in LoRaWAN. But when shadowing effects are included, it shows that with the minimized airtime (SN4) Percentage of Lost Packets are showing continuous ups and downs, which looks same like Figure 2 except for SN3.

Nonetheless, it should be measured that this accomplishment is impractical and depends on pretty hopeful guess. Firstly, a lowest amount of airtime set has a little CR set which do not give enough protection. Secondly, the slightest setting would be needed to re-examine depending on time because of the ecological changes. A set of rule might be needed to use in the LoRa arrangement to decide and regulate the settings. Nevertheless, LoRaWAN states a Network Manager element to deal purposely with this issue. The execution and its code of behaviors are not defined yet. As a consequence, LoRaWAN deployments on hand use fixed and conventional communication settings signified by SN3. [6]

In the upcoming experiment set, the impact of increasing number of sinks for each simulation run will be explored. The setting SN1 described earlier for each experimental run (one 20byte packet is transmitted by every node each $16.7 \mathrm{~min}$ and Carrier Frequency is $868 \mathrm{MHz}$ ) will be applied. Here, node positioning strategy is altered because many sinks are there now. All the Nodes are positioned in a rectangle along with a diagonal which is twice of the highest broadcast range dmax, side lengths $\mathrm{xmax}=\sqrt{3} \cdot \mathrm{dmax}$ and ymax $=$ dmax. This setup is needed as it guarantees that with communication settings SN1 nodes surrounded by this rectangle can get to minimum one sink. With four sinks or less, they are spaced evenly over xmax lying on a straight row where $y=y m a x / 2$. Either six or eight sinks are uniformly placed above the two straight lines at $\mathrm{y}=\mathrm{ymax} / 3$ and $\mathrm{y}=2 \cdot \mathrm{ymax} / 3.24$ sinks are likewise placed on three straight lines at $\mathrm{y}=\mathrm{ymax} / 4$ and $\mathrm{y}=2 \cdot \mathrm{ymax} / 4$ and $\mathrm{y}=3 \cdot \mathrm{ymax} / 4$. This sink placement strategy is chosen for simplicity rather than optimality [6].

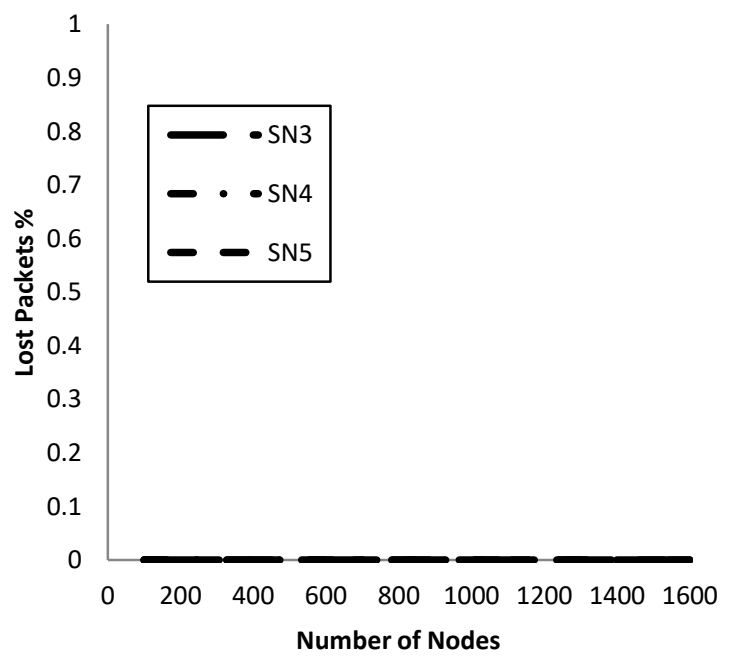

Figure 3. Lost Packets \% (without shadowing effect)

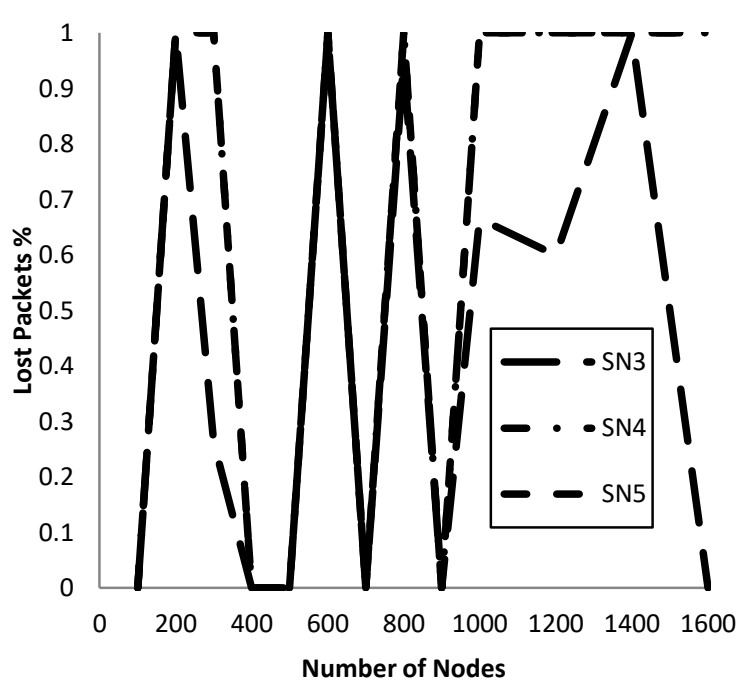

Figure 4. Lost Packets \% (with shadowing effect)

Results in Figure 5 and 6 depict the outcomes for the growing number of sinks needed as the number of nodes increased. With the increasing number of sinks, the no shadowing effect results illustrate that the percentage of lost packets is higher keeping in the process the outcome for sink 1 as " 0 ". It shows rise and drop like before with the use of shadowing effect for the increasing number of sinks and nodes. It can be deducted that this has happened because only one sink is needed where the capture effect is considered to make sure so a packet can ultimately be acknowledged. Having extra sinks, reduces the possibility for a packet to eventually find atleast one sink and not being able to steer clear of packet loss. 


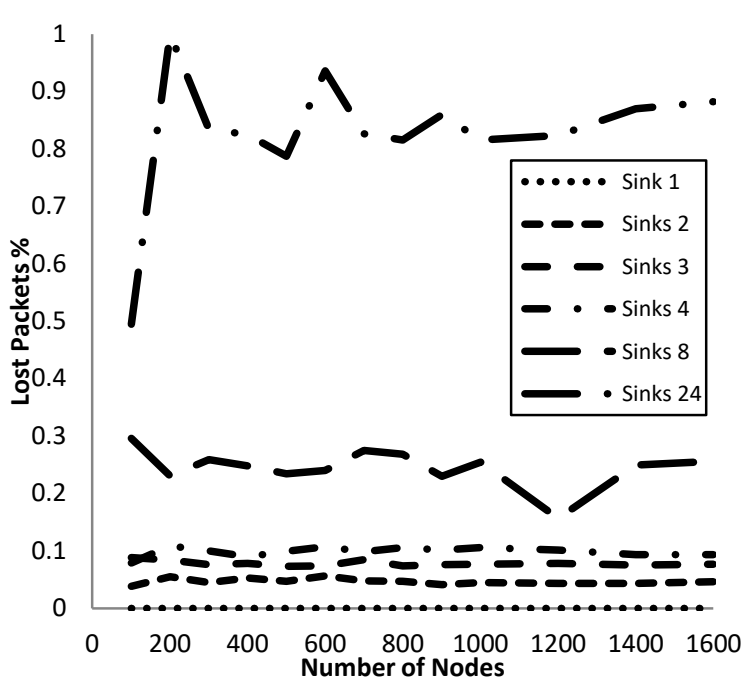

Figure 5. Lost Packets \% (without shadowing effect)

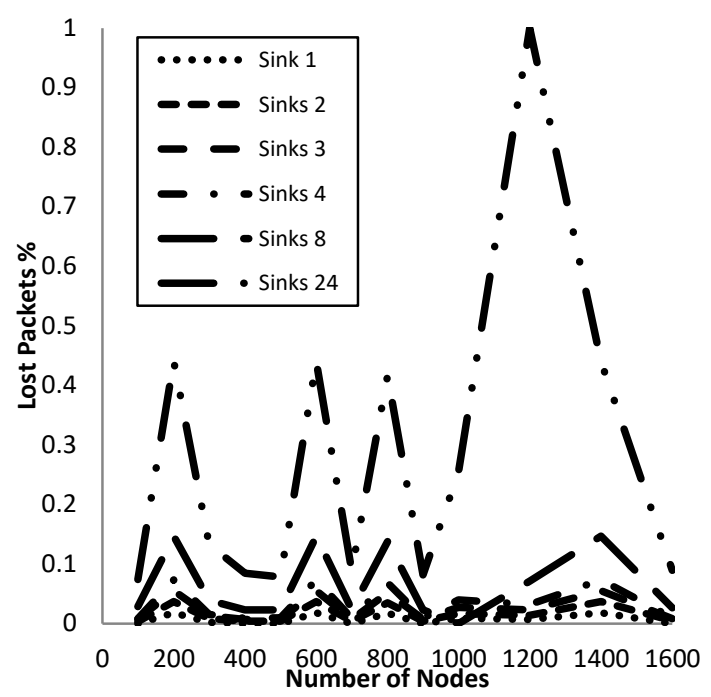

Figure 6. Lost Packets\% (with shadowing effect)

\section{CONCLUSION}

As the results presented in the paper, it is understandable that while simulated adding the shadowing effects LoRa LPWAN do not perform very well, which is not acceptable while the present world thinks about the future IoT deployments. Outcomes also show that LoRa networks cannot scale properly even with the use of dynamic transmission parameter selection and also using multiple sinks. These phases necessitate further studies because etiquettes for selecting dynamic transmission parameter and policies for functional sink employment needed to be developed for providing more useful results in near future.

\section{ACKNOWLEDGEMENTS}

This work is partially supported by International Islamic University Malaysia (IIUM) grant no. RIGS16-362-0526.

\section{REFERENCES}

[1] The Things Network. [Online]. Available: https://www.thethingsnetwork.org.

[2] Semtech. [Online]. Available: http://www.semtech.com.

[3] RF Wireless World. [Online]. Available: http://www.rfwireless-world.com.

[4] Lora alliance. [Online]. Available: http://lora-alliance.org.

[5] Semtech. AN1200.13 SX1272/3/6/7/8: LoRa Modem - Designer’s Guide, Revision 1, July 2013.

[6] Martin Bor, Utz Roedig, Thiemo Voigt, Julan M. Alonso, "Do LoRa Low-Power Wide-Area Networks Scale?", Proceedings of the 19th ACM International Conference on Modeling, Analysis and Simulation of Wireless and Mobile Systems, Pages 59-67, November 13-17, 2016.

[7] Juha Petäjäjärvi, Konstantin Mikhaylov, Antti Roivainen, Tuomo Hänninen, Marko Pettissalo, "On the Coverage of LPWANs: Range Evaluation and Channel Attenuation Model for LoRa Technology”, 14th International Conference on ITS Telecommunications (ITST), 2-4 Dec, 2015.

\section{BIOGRAPHIES OF AUTHORS}

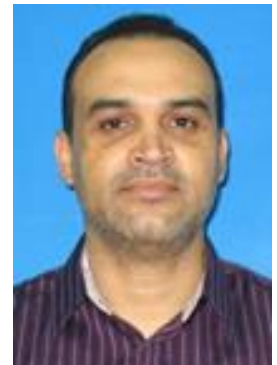

Mohamed H. Habaebi (M'99-SM'16) received his first degree from the Civil Aviation and Meteorology High Institute (CAHI), Libya ('91), his MSc in Electrical Engineering from Universiti Teknologi Malaysia ('94), and his Ph.D. in Computer and Communication System Engineering from Universiti Putra Malaysia ('01). He is currently an Associate Professor and the Post Graduate Academic Advisor at the Department of Electrical and Computer Engineering, International Islamic University Malaysia. He heads the research works on Internet of Things at the department. He has supervised many Ph.D. and M.Sc. students, published more 120 articles and papers and sits on editorial board of many international journals. He is actively publishing in M2M communication protocols, wireless sensor and actuator networks, cognitive radio, small antenna system \& radio propagation and wireless communications \& network performance 
evaluation. He is an active member of IEEE and an active reviewer to many international journals.

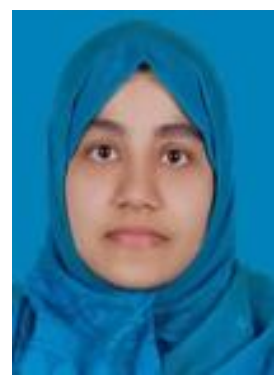

Israth Jahan Chowdhury received her Bachelor of Science in Computer Science and Engineering from International Islamic University Chittagong (IIUC) Bangladesh, in 2015 and pursuing her Master of Science in Computer and Information Engineering from International Islamic University Malaysia (IIUM). Her area of research interest is wireless communication, radio link design and Internet of Things.

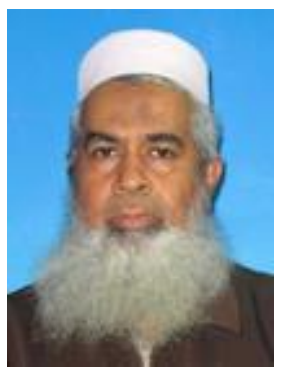

Md Rafiqul Islam (M'02) received his Bachelor of Science in Electrical and Electronic Engineering from Bangladesh University of Engineering \& Technology (BUET), Dhaka in 1987. $\mathrm{He}$ received his MSc and $\mathrm{PhD}$ both in Electrical Engineering from University of Technology Malaysia in 1996 and 2000 respectively. He is currently working as a professor in the Department of Electrical and Computer Engineering, Faculty of Engineering, International Islamic University Malaysia. He has supervised more than $50 \mathrm{PhD}$ and MSc students and has published more than 200 research papers in international journals and conferences. His areas of research interest are wireless channel modeling, radio link design, RF propagation measurement and modeling in tropical and desert, RF design, smart antennas and array antennas design, FSO propagation and modeling etc. He is a Life Fellow of Institute of Engineers Bangladesh and member of IEEE and IET.

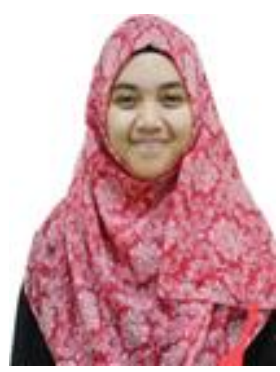

Aishah Bt. Zainal is finalizing her final year project for her BSc degree in Computer and Information Rngineering at the International Islamic Univrrsity Malaysia (IIUM). Her research interests in in Internet of Things and Wireless Communications. 